\title{
Sampled-data based consensus for multiple harmonic oscillators with directed switching topology
}

\author{
Yongfang Liu ${ }^{a, \star}$, Yu Zhao ${ }^{a}$, Zhongke Shi ${ }^{a}$ \\ ${ }^{a}$ Department of Traffic and Control Engineering, School of Automation, \\ Northwestern Polytechnical University, Xi'an Shaanxi, 710129, China
}

\begin{abstract}
This paper investigates the distributed consensus problem for a group of harmonic oscillators under directed switching topology. First, under the assumption that the union of the directed interaction graphs has a spanning tree, a class of sampleddata based consensus protocols are designed by using motion planning approaches and Pontryagin's principle. The proposed protocols only require that the union of communication topologies switched at the sampled instants has a spanning tree, without requiring information exchanges over the sampled interval and the connectivity of communication topology at each sampling instant. Then, the distributed consensus problem can be solved under directed switching topology by using stability principle and the properties of stochastic matrixes. Compared with the existing sampled-data based consensus algorithms, in this article, a remarkable advantage is that, for the proposed sampled-data based protocols, the sampled periods, communication topologies and control gains are decoupling and can be separately designed, which relaxes many restrictions in controller designs. Finally, as an extension of the leaderless consensus protocols, a leader-following consensus algorithm is further proposed in this paper. Some numerical examples are also given to illustrate the effectiveness of the analytical results.
\end{abstract}

Key words: Distributed Consensus; Sampled Data; Directed Switching Topology; Motion Planning; Harmonic Oscillator

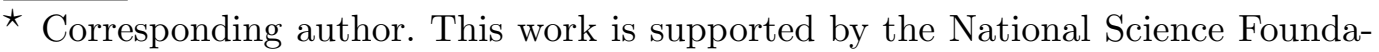
tion of China under Grants 61603300, 61603301.

Email addresses: liuyongfangpku@gmail.com (Yongfang Liu ), yuzhao5977@gmail.com (Yu Zhao), zkeshi@nwpu.edu.cn (Zhongke Shi). 


\section{Introduction}

Over the past two decades, the distributed coordination of multi-agent systems has received significant attention in the control community due to its widely applications in various engineering systems such as data fusion of sensor networks, task cooperation of robots, synchronization of distributed oscillators, and formation maneuver of unmanned vehicles. As the most fundamental research topic for multi-agent systems coordination, consensus problem has been investigated intensively, and many remarkable contributions have been made $[1-11]$.

Consensus refers to a group of agents reaching an agreement on certain quantities of interest via local interaction. By specifying desired separations among different vehicles, consensus algorithms can be applied to achieve distributed coordination including formation control and flocking. Consensus algorithms were primarily studied for multi-agent systems with single-integrator dynamics [2], where a general framework of the consensus problem was constructed. Spurred by this pioneering work [2], considerable literatures have grown up to solve the consensus problem in different scenarios, see $[4,12-15]$ and references therein. According to the absence or presence of a leader in a network, consensus can be divided into leaderless consensus and leader-following consensus. In [1], the leaderless consensus problem of first-order multi-agent systems was studied under directed topologies. Subsequently, the works $[2,3]$ extended the results of [1] to the case with dynamically changing interaction topologies. In the meanwhile, a growing interesting leaderless consensus algorithms were proposed for multi-agent systems with higher-order linear dynamics $[4,16,17]$. In some cases, it might be desirable for the states of agents to converge onto a reference trajectory. This is actually the leader-following consensus problem. Seminal algorithms for the leader-following consensus problem with linear systems $[4,21-25,20]$ were proposed to track a leader. Moreover, various leaderfollowing protocols for multiple nonlinear systems were designed in [26,29$31,19]$.

In multi-agent coordination, considering the limited bandwidth of network, it is not practical to ensure the continuity of information transmission among the neighboring agents. Thus, it is desirable to design the control protocol only using the sampled data at the discrete sampled instances rather than the whole continuous process. Many researchers have focused on consensus problems of multi-agent systems with sampled data $[32-37,18]$. By using zero-order hold circuit, the work [32] studied the consensus problems of continuous time first-order multi-agent systems via sampled control. In [34,35], sampled-data consensus algorithms for second-order systems were proposed under directed and fixed topologies. Further, consensus problem of second-order multi-agent systems was investigated by only using the sampled position information in [36]. In [37], by using past sampled position data, a novel consensus protocol was designed for second-order linear multi-agent systems with a directed communication topology. In [27], synchronization of coupled harmonic oscillators was investigated via sampled position data control. In [28], sampleddata consensus problems of linear multi-agent systems with packet losses were 
studied. It is worth noting that most of sampled-data based protocols in the above-mentioned works [32-37,27,28] were designed under conditions, which depended on the lengths of sampled periods, eigenvalues of Laplacian matrix associated to communication topologies, and control gains of the protocols. It means that the protocols are restricted under some conditions coupled with sampled periods, communication topologies and controller gains. Generally, this is too strict in practical applications. Therefore, designing sampled-data based protocols for multi-agent systems under decoupling conditions is very meaningful and significant.

Motivated by the above observations, by using motion planning approaches, this paper investigates the sampled-data based consensus problem for a group of harmonic oscillators under directed switching topologies. The main results of this paper extend the existing works on sampled-data based consensus in four aspects. (i) It is the first time that motion planning control approach is introduced to construct a new framework to solve distributed consensus problems. In this setting, for the continuous time systems considered in this paper, a novel consensus protocol is designed for each agent, which only requires the sampled measurements of the relative state information among its neighbors at sampled instants, without requiring the real-time relative state information among neighbors. So that, the networked agents only need to exchange information at the sampled instants, which greatly reduces the network communication. (ii) In this paper, the interaction topology among agents is assumed to be directed and switched, where the associated graphs may not have spanning trees. Besides, the interconnection topology only exists and switches at each sampled instant rather than sampled interval. Thus, the topology condition for achieving consensus in this paper is that the union of communication topologies switched at discrete sampled instants has a spanning tree. This extends the existing works on the case of directed interaction topology for second-order multi-agent with sampled control as studied in [34,35,37]. (iii) In many existing works $[36,37,27,28]$, the sampled periods are constrained by some conditions, which depend upon the coupling gains and communication topologies. It means that the sampled periods, communication topologies and control gains are coupled. In this paper, by using motion planing approaches, the lengths of the sampled periods can be chosen differently and arbitrarily. The communication topologies can be randomly switched under jointly spanning tree condition. Therefore, sampled periods, communication topologies and controller gains are decoupling and can be separately designed in this paper. (iv) In addition, as an extension of the above-mentioned leaderless consensus algorithm, a sampled-data based leader-following consensus protocol is proposed to solve leader-following consensus tracking problems under directed switching communication topology with a leader in networks.

The remainder of this paper is organized as follows. The preliminaries are given in Section 2. Main theoretical results are established in Sections 3 and 4. Some numerical examples are also reported in Sections 3 and 4 to illustrate the theoretical results. Concluding remarks are finally given in Section 5 . 


\section{Preliminaries}

For systems with $n$ agents, a directed graph $\mathcal{G}=(\mathcal{V}, \mathcal{E})$ is developed to model the interaction among these agents, where $\mathcal{V}=\{1,2, \cdots, N\}$ is the node set and $\mathcal{E} \subset\left\{\left(v_{i}, v_{j}\right): v_{i}, v_{j} \in \mathcal{V}\right\}$ is the edge set, where an edge is an ordered pair of vertices in $\mathcal{V}$ which means that agent $j$ can receive information from agent $i$. If there is a directed edge from $i$ to $j, i$ is defined as the parent node and $j$ is defined as the child node. The neighbors of agent $i$ are denoted by $\mathcal{N}_{i}=\left\{j \in \mathcal{V} \mid\left(v_{j}, v_{i}\right) \in \mathcal{E}\right\}$, and $\left|\mathcal{N}_{i}\right|$ is the neighbors number of agent $i$. A directed tree is a directed graph, where every node, except the root, has exactly one parent. A spanning tree of a directed graph is a directed tree formed by graph edges that connect all the nodes of the graph. We say that a graph has (or contains) a spanning tree if a subset of the edges forms a spanning tree. The interaction topologies may be dynamically changing. Therefore let $\mathcal{G}=\left\{\mathcal{G}_{1}, \mathcal{G}_{2}, \cdots, \mathcal{G}_{M}\right\}$ denote the set of all possible directed interaction graphs defined for the systems with $n$ agents. In applications, the possible interaction topologies will likely be a subset of $\mathcal{G}$. Obviously, $\mathcal{G}$ has finite elements. The union of a group of directed graphs $\left\{\mathcal{G}_{1}, \mathcal{G}_{2}, \cdots, \mathcal{G}_{M}\right\} \subset \mathcal{G}$ is a directed graph with nodes given by $\mathcal{V}$ and edge set given by the union of the edge sets of $G_{i}, i=1, \cdots, M$. The adjacency matrix $A$ associated with $\mathcal{G}$ is defined such that $a_{i j}=1$ if there is a directed edge from $j$ to $i$, and $a_{i j}=0$ otherwise. The Laplacian matrix of the graph associated with adjacency matrix $A$ is given as $\mathcal{L}=\left(l_{i j}\right)$, where $l_{i i}=\sum_{j=1}^{N} a_{i j}$ and $l_{i j}=-a_{i j}, i \neq j$.

Given $C=\left[c_{i j}\right] \in \mathbb{R}^{n \times r}$, it is said that $C \geq 0$ ( $C$ is nonnegative) if all its elements $c_{i j}$ are nonnegative, and it is said that $C>0$ ( $C$ is positive) if all its elements $c_{i j}$ are positive. Further, $C \geq D$ if $C-D \geq 0$, and $C>D$ if $C-D>0$. If a nonnegative matrix $C \in \mathbb{R}^{n \times n}$ satisfies $C \mathbf{1}=\mathbf{1}$, then it is said to be stochastic. A stochastic matrix $P$ is called indecomposable and aperiodic (SIA) if $\lim _{k \rightarrow \infty}(P)^{k}=\mathbf{1} \xi^{T}$, where $\xi$ is some column vectors.

\section{Distributed leadless consensus for harmonic oscillators under di- rected switching topology.}

In this section, sampled-data based leaderless consensus problems are investigated for multiple harmonic oscillators. Assume that there are $N$ agents in networks, which are labeled as $1,2, \cdots, N$. The behavior of these agents is described by

$$
\left\{\begin{array}{c}
\dot{p}_{i}(t)=q_{i}(t), \\
\dot{q}_{i}(t)=-\omega^{2} p_{i}(t)+u_{i}(t),
\end{array} \quad i=1,2, \cdots, N,\right.
$$


where $p_{i}(t) \in \mathbb{R}$ is the position of agent $i, q_{i}(t) \in \mathbb{R}$ its velocity vector, $u_{i}(t) \in \mathbb{R}$ its control input and $\omega$ the frequency of the oscillator.

Before moving on, the following assumption is firstly given.

Assumption 1 For a time series $\left\{t_{k}\right\}$ with $t_{0}=0$, there exist an infinite sequence of contiguous, non-empty and uniformly bounded time intervals $\left[t_{k_{j}}, t_{k_{j+1}}\right), j=$ $0,1, \cdots$, and $m_{j}$ directed switching topologies in each uniformly bounded time interval $\left[t_{k_{j}}, t_{k_{j+1}}\right)$ (i.e. there exists a finite sequence of contiguous and nonempty time subseries $\left\{t_{k_{j}}, t_{k_{j}+1}, t_{k_{j}+2}, \cdots, t_{k_{j}+m_{j}-1}\right\}$, with $t_{k_{j+1}}=t_{k_{j}+m_{j}}$, and the interconnection topologies only exist at the time instant) such that across each time interval $\left[t_{k_{j}}, t_{k_{j+1}}\right)$, the union of the directed interaction graphs at discrete times $\left\{t_{k_{j}}, t_{k_{j}+1}, t_{k_{j}+2}, \cdots, t_{k_{j}+m_{j}-1}\right\}$ has a spanning tree, where $j=0,1, \cdots, t_{k_{0}}=t_{0}$.

Lemma 1 [3] If Assumption 1 holds, then there exists a column vector $\xi$ such that

$$
\prod_{k=0}^{\infty}\left[I_{N}-\left(\mathcal{N}(k)+I_{N}\right)^{-1} \mathcal{L}(k)\right]=\mathbf{1} \xi^{T}
$$

where $\mathcal{L}(k)$ is the Laplacian matrix of the graph $\mathcal{G}(k)$ at time instant $t_{k}$, $\mathcal{N}(k)=\operatorname{diag}\left(\left|\mathcal{N}_{1}(k)\right|,\left|\mathcal{N}_{2}(k)\right|, \cdots,\left|\mathcal{N}_{N}(k)\right|\right)$, and $\left|\mathcal{N}_{i}(k)\right|$ represents the number of neighbors of agent $i$ at time instant $t_{k}$.

Definition 1 For systems (1), distributed consensus problem is said to be solved if and only if there exists a distributed protocol, based only on the relative states information such that $\lim _{t \rightarrow \infty}\left\|p_{i}(t)-p_{j}(t)\right\|=0$ and $\lim _{t \rightarrow \infty} \| q_{i}(t)-$ $q_{j}(t) \|=0$, for $i, j=1,2, \cdots, N$.

In this paper, it is assumed that only sampled relative measurements information can be used for the controller design. The main objective of this section is to design a class of sampled-data based protocols for multiple networked agents to guarantee the positions and velocities of these agents to achieve consensus.

The cost function is given by

$$
J=\frac{1}{2} \int_{t_{0}}^{t_{1}} \sum_{i=1}^{N} u_{i}^{2} d t
$$

where $t_{0}$ and $t_{1}$ are the initial and the next sampled time. The desired terminal condition at $t_{1}$ is written as

$$
p_{i}\left(t_{1}\right)=\frac{\cos \omega\left(t_{1}-t_{0}\right)}{\left|\mathcal{N}_{i}(0)\right|+1}\left[\sum_{j \in \mathcal{N}_{i}(0)} p_{j}\left(t_{0}\right)+p_{i}\left(t_{0}\right)\right]
$$




$$
\begin{aligned}
+ & \frac{\sin \omega\left(t_{1}-t_{0}\right)}{\omega\left|\mathcal{N}_{i}(0)\right|+1}\left[\sum_{j \in \mathcal{N}_{i}(0)} q_{j}\left(t_{0}\right)+q_{i}\left(t_{0}\right)\right] \\
q_{i}\left(t_{1}\right)= & \frac{-\omega \sin \omega\left(t_{1}-t_{0}\right)}{\left|\mathcal{N}_{i}(0)\right|+1}\left[\sum_{j \in \mathcal{N}_{i}(0)} p_{j}\left(t_{0}\right)+p_{i}\left(t_{0}\right)\right] \\
+ & \frac{\cos \omega\left(t_{1}-t_{0}\right)}{\left|\mathcal{N}_{i}(0)\right|+1}\left[\sum_{j \in \mathcal{N}_{i}(0)} q_{j}\left(t_{0}\right)+q_{i}\left(t_{0}\right)\right] \\
& i=1,2, \cdots, N
\end{aligned}
$$

To solve the above optimal problem, first, define the Hamiltonian function as

$$
H=\frac{1}{2} \sum_{i=1}^{N} u_{i}^{2}+\sum_{i=1}^{N}\left[\lambda_{p_{i}} q_{i}+\lambda_{q_{i}}\left(-\omega^{2} p_{i}+u_{i}\right)\right],
$$

where $\lambda_{p_{i}} \in \mathbb{R}$ and $\lambda_{q_{i}} \in \mathbb{R}$ represent the costates. Then, it follows from Pontryagin's principle that the necessary condition of optimality is written as [39]

$$
\left\{\begin{array}{l}
\dot{p}_{i}=\frac{\partial H}{\partial \lambda_{p_{i}}}=q_{i} \\
\dot{q}_{i}=-\frac{\partial H}{\partial \lambda_{q_{i}}}=-\omega^{2} p_{i}+u_{i} \\
\dot{\lambda}_{p_{i}}=-\frac{\partial H}{\partial p_{i}}=\omega^{2} \lambda_{q_{i}} \\
\dot{\lambda}_{q_{i}}=-\frac{\partial H}{\partial q_{i}}=-\lambda_{p_{i}}
\end{array}\right.
$$

Besides, according to the extremal condition

$$
\frac{\partial H}{\partial u_{i}}=u_{i}+\lambda_{q_{i}}=0
$$

it follows that the finite-time optimal motion-planning control for systems (1) is

$$
u_{i}=-\lambda_{q_{i}}
$$

By integrating $(5 \mathrm{c})$ and $(5 \mathrm{~d})$ from $t_{0}$ to $t$, one has

$$
\begin{aligned}
& \lambda_{p_{i}}(t)=\cos \omega\left(t-t_{0}\right) \lambda_{p_{i}}\left(t_{0}\right)+\omega \sin \omega\left(t-t_{0}\right) \lambda_{q_{i}}\left(t_{0}\right), \\
& \lambda_{q_{i}}(t)=-\frac{1}{\omega} \sin \omega\left(t-t_{0}\right) \lambda_{p_{i}}\left(t_{0}\right)+\cos \omega\left(t-t_{0}\right) \lambda_{q_{i}}\left(t_{0}\right) .
\end{aligned}
$$

Thus, it is followed (6) that 


$$
u_{i}=-\lambda_{q_{i}}=\frac{1}{\omega} \sin \omega\left(t-t_{0}\right) \lambda_{p_{i}}\left(t_{0}\right)-\cos \omega\left(t-t_{0}\right) \lambda_{q_{i}}\left(t_{0}\right),
$$

where $\lambda_{p_{i}}\left(t_{0}\right)$ and $\lambda_{p_{i}}\left(t_{0}\right)$ are the initial states of $\lambda_{p_{i}}$ and $\lambda_{p_{i}}$, respectively. Then, the determination of the optimal control (6) is boiled down to finding the initial states of $\lambda_{p_{i}}$ and $\lambda_{p_{i}}$. By substituting (7) into (5b) and integrating (5a) and (5b) from $t_{0}$ to $t_{1}$, one gets

$$
\begin{aligned}
p_{i}\left(t_{1}\right)= & \cos \omega\left(t_{1}-t_{0}\right) p_{i}\left(t_{0}\right)+\frac{1}{\omega} \sin \omega\left(t_{1}-t_{0}\right) q_{i}\left(t_{0}\right) \\
& +\int_{t_{0}}^{t_{1}} \frac{1}{\omega^{2}} \sin \omega\left(t_{1}-\tau\right) \sin \omega\left(\tau-t_{0}\right) d \tau \cdot \lambda_{p_{i}}\left(t_{0}\right) \\
& +\int_{t_{0}}^{t_{1}}-\frac{1}{\omega} \sin \omega\left(t_{1}-\tau\right) \cos \omega\left(\tau-t_{0}\right) d \tau \cdot \lambda_{q_{i}}\left(t_{0}\right) \\
= & \cos \omega\left(t_{1}-t_{0}\right) p_{i}\left(t_{0}\right)+\frac{1}{\omega} \sin \omega\left(t_{1}-t_{0}\right) q_{i}\left(t_{0}\right) \\
& +\left[\frac{\sin \omega\left(t_{1}-t_{0}\right)}{2 \omega^{3}}-\frac{t_{1}-t_{0}}{2 \omega^{2}} \cos \omega\left(t_{1}-t_{0}\right)\right] \lambda_{p_{i}}\left(t_{0}\right) \\
& -\frac{t_{1}-t_{0}}{2 \omega} \sin \omega\left(t_{1}-t_{0}\right) \lambda_{q_{i}}\left(t_{0}\right), \\
q_{i}\left(t_{1}\right)= & -\omega \sin \omega\left(t_{1}-t_{0}\right) p_{i}\left(t_{0}\right)+\cos \omega\left(t_{1}-t_{0}\right) q_{i}\left(t_{0}\right) \\
& +\int_{t_{0}}^{t_{1}} \frac{1}{\omega} \cos \omega\left(t_{1}-\tau\right) \sin \omega\left(\tau-t_{0}\right) d \tau \cdot \lambda_{p_{i}}\left(t_{0}\right) \\
& +\int_{t_{0}}^{t_{1}}-\cos \omega\left(t_{1}-\tau\right) \cos \omega\left(\tau-t_{0}\right) d \tau \cdot \lambda_{q_{i}}\left(t_{0}\right) \\
= & -\omega \sin \omega\left(t_{1}-t_{0}\right) p_{i}\left(t_{0}\right)+\cos \omega\left(t_{1}-t_{0}\right) q_{i}\left(t_{0}\right) \\
& +\frac{t_{1}-t_{0}}{2 \omega} \sin \omega\left(t_{1}-t_{0}\right) \lambda_{p_{i}}\left(t_{0}\right) \\
& -\left[\frac{\sin \omega\left(t_{1}-t_{0}\right)}{2 \omega}+\frac{t_{1}-t_{0}}{2} \cos \omega\left(t_{1}-t_{0}\right)\right] \lambda_{q_{i}}\left(t_{0}\right) .
\end{aligned}
$$

Note that for $t_{1}>t_{0}, \sin ^{2}\left(t_{1}-t_{0}\right)-\left(t_{1}-t_{0}\right)^{2} \neq 0$. Considering the terminal condition (3), it is followed from the above equations that

$$
\begin{aligned}
\lambda_{p_{i}}\left(t_{0}\right)= & \frac{2 \omega^{4}\left(t_{1}-t_{0}\right)+2 \omega^{3} \sin \omega\left(t_{1}-t_{0}\right) \cos \omega\left(t_{1}-t_{0}\right)}{\sin ^{2} \omega\left(t_{1}-t_{0}\right)-\omega^{2}\left(t_{1}-t_{0}\right)^{2}} \\
& \cdot \frac{1}{\left|\mathcal{N}_{i}(0)\right|+1} \sum_{j \in \mathcal{N}_{i}(0)}\left[p_{j}\left(t_{0}\right)-p_{i}\left(t_{0}\right)\right] \\
& +\frac{2 \omega^{3} \sin ^{2} \omega\left(t_{1}-t_{0}\right)}{\sin ^{2} \omega\left(t_{1}-t_{0}\right)-\omega^{2}\left(t_{1}-t_{0}\right)^{2}}
\end{aligned}
$$




$$
\begin{aligned}
& \frac{1}{\left|\mathcal{N}_{i}(0)\right|+1} \sum_{j \in \mathcal{N}_{i}(0)}\left[q_{j}\left(t_{0}\right)-q_{i}\left(t_{0}\right)\right] \\
& \lambda_{q_{i}}\left(t_{0}\right)=\frac{2 \omega^{3} \sin ^{2} \omega\left(t_{1}-t_{0}\right)}{\sin ^{2} \omega\left(t_{1}-t_{0}\right)-\omega^{2}\left(t_{1}-t_{0}\right)^{2}} \\
& \cdot \frac{1}{\left|\mathcal{N}_{i}(0)\right|+1} \sum_{j \in \mathcal{N}_{i}(0)}\left[p_{j}\left(t_{0}\right)-p_{i}\left(t_{0}\right)\right] \\
& +\frac{2 \omega^{2}\left(t_{1}-t_{0}\right)-2 \omega \sin \omega\left(t_{1}-t_{0}\right) \cos \omega\left(t_{1}-t_{0}\right)}{\sin ^{2} \omega\left(t_{1}-t_{0}\right)-\omega^{2}\left(t_{1}-t_{0}\right)^{2}} \\
& \frac{1}{\left|\mathcal{N}_{i}(0)\right|+1} \sum_{j \in \mathcal{N}_{i}(0)}\left[q_{j}\left(t_{0}\right)-q_{i}\left(t_{0}\right)\right] \text {. }
\end{aligned}
$$

Thus, the optimal control (6) is given by (7), (8) and (9). So, to summarize so far, with control law (6), systems (1) is derived from $p_{i}\left(t_{0}\right), q_{i}\left(t_{0}\right) i=$ $1,2, \cdots, N$ to the terminal conditions (3). That is to say, with control law (6), every agent in systems (1) is derived to the predicted average states of all neighbors. From an intuitional point of view, after several times such motion planning, agents in systems (1) will achieve consensus. Therefore, select a time series $\left\{t_{k}=t_{0}+T_{k}\right\}$, where $T_{k}>0$ is bounded and given according to task requirements. Based only onthe sampled measurement information, we propose the following consensus protocol:

$$
\begin{aligned}
u_{i}(t)= & \frac{\alpha\left(t, t_{k}, t_{k+1}\right)}{\left|\mathcal{N}_{i}(k)\right|+1} \sum_{j \in \mathcal{N}_{i}(k)} a_{i j}(k)\left[p_{j}\left(t_{k}\right)-p_{i}\left(t_{k}\right)\right] \\
& +\frac{\beta\left(t, t_{k}, t_{k+1}\right)}{\left|\mathcal{N}_{i}(k)\right|+1} \sum_{j \in \mathcal{N}_{i}(k)} a_{i j}(k)\left[q_{j}\left(t_{k}\right)-q_{i}\left(t_{k}\right)\right],
\end{aligned}
$$

where $t_{k} \leq t<t_{k+1}, k=0,1, \cdots, a_{i j}(k)$ is the $(i, j)$ th entry of the time-varying adjacency matrix $A(k)$ at sampled time $t_{k}$, and control gain $\alpha\left(t, t_{k}, t_{k+1}\right)$, $\beta\left(t, t_{k}, t_{k+1}\right)$ satisfy

$$
\begin{aligned}
\alpha\left(t, t_{k}, t_{k+1}\right)= & \left\{\frac{2 \omega^{4}\left(t_{k+1}-t_{k}\right)}{\omega\left(\sin ^{2}\left[\omega\left(t_{k+1}-t_{k}\right)\right]-\omega^{2}\left(t_{k+1}-t_{k}\right)^{2}\right)}\right. \\
& \left.+\frac{2 \omega^{3} \sin \left[\omega\left(t_{k+1}-t_{k}\right)\right] \cos \left[\omega\left(t_{k+1}-t_{k}\right)\right]}{\omega\left(\sin ^{2}\left[\omega\left(t_{k+1}-t_{k}\right)\right]-\omega^{2}\left(t_{k+1}-t_{k}\right)^{2}\right)}\right\} \\
& \cdot \sin \left[\omega\left(t-t_{k}\right)\right]-\frac{2 \omega^{3} \sin ^{2}\left[\omega\left(t_{k+1}-t_{k}\right)\right] \cos \left[\omega\left(t-t_{k}\right)\right]}{\sin ^{2}\left[\omega\left(t_{k+1}-t_{k}\right)\right]-\omega^{2}\left(t_{k+1}-t_{k}\right)^{2}} \\
\beta\left(t, t_{k}, t_{k+1}\right)= & \frac{2 \omega^{3} \sin ^{2}\left[\omega\left(t_{k+1}-t_{k}\right)\right] \sin \left[\omega\left(t-t_{k}\right)\right]}{\omega\left(\sin ^{2}\left[\omega\left(t_{k+1}-t_{k}\right)\right]-\omega^{2}\left(t_{k+1}-t_{k}\right)^{2}\right)} \\
& -\left\{\frac{2 \omega^{2}\left(t_{k+1}-t_{k}\right)}{\sin ^{2}\left[\omega\left(t_{k+1}-t_{k}\right)\right]-\omega^{2}\left(t_{k+1}-t_{k}\right)^{2}}\right.
\end{aligned}
$$




$$
\left.-\frac{2 \omega \sin \left[\omega\left(t_{k+1}-t_{k}\right)\right] \cos \left[\omega\left(t_{k+1}-t_{k}\right)\right]}{\sin ^{2}\left[\omega\left(t_{k+1}-t_{k}\right)\right]-\omega^{2}\left(t_{k+1}-t_{k}\right)^{2}}\right\} \cdot \cos \left[\omega\left(t-t_{k}\right)\right]
$$

Theorem 1 Suppose Assumption 1 holds. For the given time series $\left\{t_{k}, k=\right.$ $0,1, \cdots\}$ satisfying $0<T_{l} \leq t_{k+1}-t_{k} \leq T_{u}<\infty$, where $T_{l}$ and $T_{u}$ are two arbitrary positive constants, distributed consensus problem for multi-agent systems (1) is solved by using the sampled-data based protocol (10). Furthermore, the final consensus value is given by

$$
\begin{aligned}
& p^{*}(t)=\cos \left(t-t_{0}\right) \sum_{i=1}^{N} \xi_{i} p_{i}\left(t_{0}\right)+\frac{1}{\omega} \sin \left(t-t_{0}\right) \sum_{i=1}^{N} \xi_{i} q_{i}\left(t_{0}\right), \\
& q^{*}(t)=-\omega \sin \left(t-t_{0}\right) \sum_{i=1}^{N} \xi_{i} p_{i}\left(t_{0}\right)+\cos \left(t-t_{0}\right) \sum_{i=1}^{N} \xi_{i} q_{i}\left(t_{0}\right) .
\end{aligned}
$$

where $\xi_{i}$ is the ith element of vector $\xi$.

Proof: First, it is to prove that at the sampled time series $\left\{t_{k}\right\}$, the states of systems (1) with (10) will achieve consensus. Substitute (10) into (1), one gets

$$
\begin{aligned}
\dot{p}_{i}(t)= & q_{i}(t), \\
\dot{q}_{i}(t)= & -\omega^{2} p_{i}(t)+\frac{\alpha\left(t, t_{k}, t_{k+1}\right)}{\left|\mathcal{N}_{i}(k)\right|+1} \sum_{j \in \mathcal{N}_{i}(k)} a_{i j}(k)\left[p_{j}\left(t_{k}\right)-p_{i}\left(t_{k}\right)\right] \\
& +\frac{\beta\left(t, t_{k}, t_{k+1}\right)}{\left|\mathcal{N}_{i}(k)\right|+1} \sum_{j \in \mathcal{N}_{i}(k)} a_{i j}(k)\left[q_{j}\left(t_{k}\right)-q_{i}\left(t_{k}\right)\right],
\end{aligned}
$$

where $\alpha\left(t, t_{k}, t_{k+1}\right)$ and $\beta\left(t, t_{k}, t_{k+1}\right)$ are given in (11). Then, by integrating (12) from $t_{k}$ to $t_{k+1}, k=0,1, \cdots$, it is obtained that

$$
\begin{aligned}
p_{i}\left(t_{k+1}\right)= & \frac{\cos \left[\omega\left(t_{k+1}-t_{k}\right)\right]}{\left|\mathcal{N}_{i}(k)\right|+1}\left[\sum_{j \in \mathcal{N}_{i}(k)} a_{i j}(k) p_{j}\left(t_{k}\right)+p_{i}\left(t_{k}\right)\right] \\
& +\frac{\sin \left[\omega\left(t_{k+1}-t_{k}\right)\right]}{\omega\left|\mathcal{N}_{i}(k)\right|+1}\left[\sum_{j \in \mathcal{N}_{i}(k)} a_{i j}(k) q_{j}\left(t_{k}\right)+q_{i}\left(t_{k}\right)\right], \\
q_{i}\left(t_{k+1}\right)= & \frac{-\omega \sin \omega\left(t_{k+1}-t_{k}\right)}{\left|\mathcal{N}_{i}(k)\right|+1}\left[\sum_{j \in \mathcal{N}_{i}(k)} a_{i j}(k) p_{j}\left(t_{k}\right)+p_{i}\left(t_{k}\right)\right] \\
+ & \frac{\cos \left[\omega\left(t_{k+1}-t_{k}\right)\right]}{\left|\mathcal{N}_{i}(k)\right|+1}\left[\sum_{j \in \mathcal{N}_{i}(k)} a_{i j}(k) q_{j}\left(t_{k}\right)+q_{i}\left(t_{k}\right)\right], \\
& i=1,2, \cdots, N .
\end{aligned}
$$

Further, it is followed that 


$$
\begin{aligned}
& p_{i}\left(t_{k+1}\right)-\cos \left[\omega\left(t_{k+1}-t_{k}\right)\right] p_{i}\left(t_{k}\right)-\frac{1}{\omega} \sin \left[\omega\left(t_{k+1}-t_{k}\right)\right] q_{i}\left(t_{k}\right) \\
= & \frac{\cos \left[\omega\left(t_{k+1}-t_{k}\right)\right]}{\left|\mathcal{N}_{i}(k)\right|+1} \sum_{j \in \mathcal{N}_{i}(k)} a_{i j}(k)\left[p_{j}\left(t_{k}\right)-p_{i}\left(t_{k}\right)\right] \\
& +\frac{\sin \left[\omega\left(t_{k+1}-t_{k}\right)\right]}{\omega\left|\mathcal{N}_{i}(k)\right|+1} \sum_{j \in \mathcal{N}_{i}(k)} a_{i j}(k)\left[q_{j}\left(t_{k}\right)-q_{i}\left(t_{k}\right)\right], \\
& q_{i}\left(t_{k+1}\right)+\omega \sin \left[\omega\left(t_{k+1}-t_{k}\right)\right] p_{i}\left(t_{k}\right)-\cos \left[\omega\left(t_{k+1}-t_{k}\right)\right] q_{i}\left(t_{k}\right) \\
= & -\frac{\omega \sin \left[\omega\left(t_{k+1}-t_{k}\right)\right]}{\left|\mathcal{N}_{i}(k)\right|+1} \sum_{j \in \mathcal{N}_{i}(k)} a_{i j}(k)\left[p_{j}\left(t_{k}\right)-p_{i}\left(t_{k}\right)\right] \\
& +\frac{\cos \left[\omega\left(t_{k+1}-t_{k}\right)\right]}{\left|\mathcal{N}_{i}(k)\right|+1} \sum_{j \in \mathcal{N}_{i}(k)} a_{i j}(k)\left[q_{j}\left(t_{k}\right)-q_{i}\left(t_{k}\right)\right] .
\end{aligned}
$$

Let $P\left(t_{k}\right)=\left(p_{1}\left(t_{k}\right), p_{2}\left(t_{k}\right), \cdots, p_{N}\left(t_{k}\right)\right)^{T}$ and $Q\left(t_{k}\right)=\left(q_{1}\left(t_{k}\right), q_{2}\left(t_{k}\right), \cdots, q_{N}\left(t_{k}\right)\right)^{T}$. It follows that,

$$
\begin{aligned}
P\left(t_{k+1}\right)= & \cos \left[\omega\left(t_{k+1}-t_{k}\right)\right]\left(I_{N}-\left(\mathcal{N}(k)+I_{N}\right)^{-1} \mathcal{L}(k)\right) \cdot P\left(t_{k}\right) \\
& +\frac{1}{\omega} \sin \left[\omega\left(t_{k+1}-t_{k}\right)\right]\left(I_{N}-\left(\mathcal{N}(k)+I_{N}\right)^{-1} \mathcal{L}(k)\right) \cdot Q\left(t_{k}\right), \\
Q\left(t_{k+1}\right)= & -\omega \sin \left[\omega\left(t_{k+1}-t_{k}\right)\right]\left(I_{N}-\left(\mathcal{N}(k)+I_{N}\right)^{-1} \mathcal{L}(k)\right) \cdot P\left(t_{k}\right) \\
& +\cos \left[\omega\left(t_{k+1}-t_{k}\right)\right]\left(I_{N}-\left(\mathcal{N}(k)+I_{N}\right)^{-1} \mathcal{L}(k)\right) \cdot Q\left(t_{k}\right),
\end{aligned}
$$

where $\mathcal{N}(k)=\operatorname{diag}\left(\left|\mathcal{N}_{1}(k)\right|,\left|\mathcal{N}_{2}(k)\right|, \cdots,\left|\mathcal{N}_{N}(k)\right|\right)$. In the matrix form, one gets

$$
\begin{aligned}
\left(\begin{array}{l}
P\left(t_{k+1}\right) \\
Q\left(t_{k+1}\right)
\end{array}\right)= & \left(\begin{array}{cc}
\cos \left[\omega\left(t_{k+1}-t_{k}\right)\right] & \frac{1}{\omega} \sin \left[\omega\left(t_{k+1}-t_{k}\right)\right] \\
-\omega \sin \left[\omega\left(t_{k+1}-t_{k}\right)\right] & \cos \left[\omega\left(t_{k+1}-t_{k}\right)\right]
\end{array}\right) \\
& \otimes\left(I_{N}-\left(\mathcal{N}(k)+I_{N}\right)^{-1} \mathcal{L}(k)\right)\left(\begin{array}{c}
P\left(t_{k}\right) \\
Q\left(t_{k}\right)
\end{array}\right) \\
= & \left(\begin{array}{cc}
\cos \omega\left(t_{k+1}-t_{0}\right) & \frac{1}{\omega} \sin \omega\left(t_{k+1}-t_{0}\right) \\
-\omega \sin \omega\left(t_{k+1}-t_{0}\right) & \cos \omega\left(t_{k+1}-t_{0}\right)
\end{array}\right) \\
& \otimes \prod_{s=0}^{k}\left(I_{N}-\left(\mathcal{N}(s)+I_{N}\right)^{-1} \mathcal{L}(s)\right)\left(\begin{array}{c}
P\left(t_{0}\right) \\
Q\left(t_{0}\right)
\end{array}\right) .
\end{aligned}
$$

Let $P^{*}(t)=\mathbf{1} p^{*}(t)$ and $Q^{*}(t)=\mathbf{1} q^{*}(t)$, one has

$$
\left(\begin{array}{l}
P^{*}(t) \\
Q^{*}(t)
\end{array}\right)=\left(\begin{array}{cc}
\cos \left[\omega\left(t-t_{0}\right)\right] & \frac{1}{\omega} \sin \left[\omega\left(t-t_{0}\right)\right] \\
-\omega \sin \left[\omega\left(t-t_{0}\right)\right] & \cos \left[\omega\left(t-t_{0}\right)\right]
\end{array}\right) \otimes\left(\mathbf{1} \xi^{T}\right)\left(\begin{array}{c}
P\left(t_{0}\right) \\
Q\left(t_{0}\right)
\end{array}\right)
$$


Then, one gets

$$
\begin{aligned}
& \lim _{k \rightarrow \infty}\left[\left(\begin{array}{l}
P\left(t_{k+1}\right) \\
Q\left(t_{k+1}\right)
\end{array}\right)-\left(\begin{array}{c}
P^{*}\left(t_{k+1}\right) \\
Q^{*}\left(t_{k+1}\right)
\end{array}\right)\right] \\
& =\lim _{k \rightarrow \infty}\left(\begin{array}{cc}
\cos \left[\omega\left(t_{k+1}-t_{0}\right)\right] & \frac{1}{\omega} \sin \left[\omega\left(t_{k+1}-t_{0}\right)\right] \\
-\omega \sin \left[\omega\left(t_{k+1}-t_{0}\right)\right] & \cos \left[\omega\left(t_{k+1}-t_{0}\right)\right]
\end{array}\right) \\
& \otimes\left(\prod_{s=0}^{k}\left(I_{N}-\left(\mathcal{N}(s)+I_{N}\right)^{-1} \mathcal{L}(s)\right)-\mathbf{1} \xi^{T}\right)\left(\begin{array}{c}
P\left(t_{0}\right) \\
Q\left(t_{0}\right)
\end{array}\right) .
\end{aligned}
$$

Under Assumption 1, one has that the union of the directed switching interaction graphs has a spanning tree. It follows from Lemma 1 that $\lim _{k \rightarrow \infty} \prod_{s=0}^{k}\left(I_{N}-\right.$ $\left.\left(\mathcal{N}(s)+I_{N}\right)^{-1} \mathcal{L}(s)\right)=\mathbf{1} \xi^{T}$ for some column vector $\xi$. Since each entry of

$$
\left(\begin{array}{cc}
\cos \left[\omega\left(t_{k+1}-t_{0}\right)\right] & \frac{1}{\omega} \sin \left[\omega\left(t_{k+1}-t_{0}\right)\right] \\
-\omega \sin \left[\omega\left(t_{k+1}-t_{0}\right)\right] & \cos \left[\omega\left(t_{k+1}-t_{0}\right)\right]
\end{array}\right)
$$

is bounded, one has

$$
\lim _{k \rightarrow \infty}\left[\left(\begin{array}{l}
P\left(t_{k}\right) \\
Q\left(t_{k}\right)
\end{array}\right)-\left(\begin{array}{l}
P^{*}\left(t_{k}\right) \\
Q^{*}\left(t_{k}\right)
\end{array}\right)\right]=0 .
$$

Note that

$$
\begin{aligned}
& P^{*}\left(t_{k}\right)=\left[\cos \left[\omega\left(t_{k}-t_{0}\right)\right] \xi^{T} P\left(t_{0}\right)+\frac{1}{\omega} \sin \left[\omega\left(t_{k}-t_{0}\right)\right] \xi^{T} Q\left(t_{0}\right)\right] \cdot \mathbf{1}, \\
& Q^{*}\left(t_{k}\right)=\left[-\omega \sin \left[\omega\left(t_{k}-t_{0}\right)\right] \xi^{T} P\left(t_{0}\right)+\cos \left[\omega\left(t_{k}-t_{0}\right)\right] \xi^{T} Q\left(t_{0}\right)\right] \cdot \mathbf{1} .
\end{aligned}
$$

Thus, it is obtained that $\lim _{k \rightarrow \infty}\left\|p_{i}\left(t_{k}\right)-p_{j}\left(t_{k}\right)\right\|=0, \lim _{k \rightarrow \infty} \| q_{i}\left(t_{k}\right)-$ $q_{j}\left(t_{k}\right) \|=0, \quad i, j=1,2, \cdots, N$.

Then, it is to prove that consensus will achieve with time evolution. Integrate the closed-systems (12) from $t_{k}$ to $t$, where $t_{k+1} \leq t<t_{k}$. It is obtained that

$$
\begin{aligned}
p_{i}(t)= & \cos \left[\omega\left(t-t_{k}\right)\right] p_{i}\left(t_{k}\right)+\frac{1}{\omega} \sin \left[\omega\left(t-t_{k}\right)\right] q_{i}\left(t_{k}\right) \\
& +\left[\frac{\sin \left[\omega\left(t-t_{k}\right)\right]}{2 \omega^{3}}-\frac{t-t_{k}}{2 \omega^{2}} \cos \left[\omega\left(t-t_{k}\right)\right]\right] \lambda_{p_{i}}\left(t_{k}\right) \\
& -\frac{t-t_{k}}{2 \omega} \sin \left[\omega\left(t-t_{k}\right)\right] \lambda_{q_{i}}\left(t_{k}\right), t_{k} \leq t<t_{k+1},
\end{aligned}
$$

where 


$$
\begin{aligned}
\lambda_{p_{i}}\left(t_{k}\right)= & \left\{\frac{2 \omega^{4}\left(t_{k+1}-t_{k}\right)}{\sin ^{2}\left[\omega\left(t_{k+1}-t_{k}\right)\right]-\omega^{2}\left(t_{k+1}-t_{k}\right)^{2}}\right. \\
& \left.+\frac{2 \omega^{3} \sin \left[\omega\left(t_{k+1}-t_{k}\right)\right] \cos \left[\omega\left(t_{k+1}-t_{k}\right)\right]}{\sin ^{2}\left[\omega\left(t_{k+1}-t_{k}\right)\right]-\omega^{2}\left(t_{k+1}-t_{k}\right)^{2}}\right\} \\
& \cdot \frac{1}{\left|\mathcal{N}_{i}(k)\right|+1} \sum_{j \in \mathcal{N}_{i}(k)} a_{i j}(k)\left[p_{j}\left(t_{k}\right)-p_{i}\left(t_{k}\right)\right] \\
& +\left\{\frac{2 \omega^{3} \sin ^{2}\left[\omega\left(t_{k+1}-t_{k}\right)\right]}{\sin ^{2}\left[\omega\left(t_{k+1}-t_{k}\right)\right]-\omega^{2}\left(t_{k+1}-t_{k}\right)^{2}}\right\} \\
& \cdot \frac{1}{\left|\mathcal{N}_{i}(k)\right|+1} \sum_{j \in \mathcal{N}_{i}(k)} a_{i j}(k)\left[q_{j}\left(t_{k}\right)-q_{i}\left(t_{k}\right)\right], \\
\lambda_{q_{i}}\left(t_{k}\right)= & \frac{2 \omega^{3} \sin ^{2}\left[\omega\left(t_{k+1}-t_{k}\right)\right]}{\sin ^{2}\left[\omega\left(t_{k+1}-t_{k}\right)\right]-\omega^{2}\left(t_{k+1}-t_{k}\right)^{2}} \\
& \cdot \frac{1}{\left|\mathcal{N}_{i}(k)\right|+1} \sum_{j \in \mathcal{N}_{i}(k)} a_{i j}(k) a_{i j}(k)\left[p_{j}\left(t_{k}\right)-p_{i}\left(t_{k}\right)\right] \\
& +\left\{\frac{2 \omega^{2}\left(t_{k+1}-t_{k}\right)}{\sin ^{2}\left[\omega\left(t_{k+1}-t_{k}\right)\right]-\omega^{2}\left(t_{k+1}-t_{k}\right)^{2}}\right. \\
& \left.-\frac{2 \omega \sin ^{2}\left[\omega\left(t_{k+1}-t_{k}\right)\right] \cos \left[\omega\left(t_{k+1}-t_{k}\right)\right]}{\sin ^{2}\left[\omega\left(t_{k+1}-t_{k}\right)\right]-\omega^{2}\left(t_{k+1}-t_{k}\right)^{2}}\right\} \\
& \cdot \frac{1}{\left|\mathcal{N}_{i}(k)\right|+1} \sum_{j \in \mathcal{N}_{i}(k)} a_{i j}(k)\left[q_{j}\left(t_{k}\right)-q_{i}\left(t_{k}\right)\right] .
\end{aligned}
$$

Thus, one has

$$
\begin{aligned}
p_{i}(t)-p_{j}(t)= & \cos \left[\omega\left(t-t_{k}\right)\right]\left(p_{i}\left(t_{k}\right)-p_{j}\left(t_{k}\right)\right) \\
& +\frac{1}{\omega} \sin \left[\omega\left(t-t_{k}\right)\right]\left(q_{i}\left(t_{k}\right)-q_{j}\left(t_{k}\right)\right) \\
& +\left[\frac{\sin \left[\omega\left(t-t_{k}\right)\right]}{2 \omega^{3}}-\frac{t-t_{k}}{2 \omega^{2}} \cos \left[\omega\left(t-t_{k}\right)\right]\right] \lambda_{p_{i}}\left(t_{k}\right) \\
& -\frac{t-t_{k}}{2 \omega} \sin \left[\omega\left(t-t_{k}\right)\right] \lambda_{q_{i}}\left(t_{k}\right) \\
& -\left[\frac{\sin \left[\omega\left(t-t_{k}\right)\right]}{2 \omega^{3}}-\frac{t-t_{k}}{2 \omega^{2}} \cos \left[\omega\left(t-t_{k}\right)\right]\right] \lambda_{p_{j}}\left(t_{k}\right) \\
& +\frac{t-t_{k}}{2 \omega} \sin \left[\omega\left(t-t_{k}\right)\right] \lambda_{q_{j}}\left(t_{k}\right) .
\end{aligned}
$$

Further,

$$
\begin{aligned}
\left\|p_{i}(t)-p_{j}(t)\right\| \leq & \left\|p_{i}\left(t_{k}\right)-p_{j}\left(t_{k}\right)\right\|+\frac{1}{\omega}\left\|q_{i}\left(t_{k}\right)-q_{j}\left(t_{k}\right)\right\| \\
& +\frac{1+\left[\omega\left(t_{k+1}-t_{k}\right)\right]}{2 \omega^{3}}\left\|\lambda_{p_{i}}\left(t_{k}\right)\right\|
\end{aligned}
$$




$$
\begin{aligned}
& +\frac{t_{k+1}-t_{k}}{2 \omega}\left\|\lambda_{q_{i}}\left(t_{k}\right)\right\|+\frac{1+\left[\omega\left(t_{k+1}-t_{k}\right)\right]}{2 \omega^{3}}\left\|\lambda_{p_{j}}\left(t_{k}\right)\right\| \\
& +\frac{t_{k+1}-t_{k}}{2 \omega}\left\|\lambda_{q_{j}}\left(t_{k}\right)\right\| .
\end{aligned}
$$

Considering (13), one gets

$$
\begin{aligned}
\left\|\lambda_{p_{i}}\left(t_{k}\right)\right\| \leq & \frac{2 \omega^{4}\left(t_{k+1}-t_{k}\right)+2 \omega^{3}}{\omega^{2}\left(t_{k+1}-t_{k}\right)^{2}-\sin ^{2}\left[\omega\left(t_{k+1}-t_{k}\right)\right]} \\
& \cdot \frac{1}{\left|\mathcal{N}_{i}(k)\right|+1} \sum_{j \in \mathcal{N}_{i}(k)}\left\|p_{j}\left(t_{k}\right)-p_{i}\left(t_{k}\right)\right\| \\
& +\frac{2 \omega^{3}}{\omega^{2}\left(t_{k+1}-t_{k}\right)^{2}-\sin ^{2}\left[\omega\left(t_{k+1}-t_{k}\right)\right]} \\
& \cdot \frac{1}{\left|\mathcal{N}_{i}(k)\right|+1} \sum_{j \in \mathcal{N}_{i}(k)}\left\|q_{j}\left(t_{k}\right)-q_{i}\left(t_{k}\right)\right\| .
\end{aligned}
$$

Noting that $0<T_{l} \leq t_{k+1}-t_{k} \leq T_{u}<\infty, \lim _{k \rightarrow \infty}\left\|p_{i}\left(t_{k}\right)-p_{j}\left(t_{k}\right)\right\|=0$ and $\lim _{k \rightarrow \infty}\left\|q_{i}\left(t_{k}\right)-q_{j}\left(t_{k}\right)\right\|=0, i, \bar{j}=1,2, \cdots, N$, one gets

$$
\lim _{t \rightarrow \infty}\left\|p_{i}(t)-p_{j}(t)\right\|=0, t_{k} \leq t<t_{k+1}
$$

Similarly, it is followed that

$$
\lim _{t \rightarrow \infty}\left\|q_{i}(t)-q_{j}(t)\right\|=0, t_{k} \leq t<t_{k+1} .
$$

Therefore, under the protocol (10), multi-agent systems (1) can achieve consensus. The proof is completed.

Remark 1 By using motion planning approaches, in this paper, a new framework is presented to solve the consensus problem of multi-agent systems. In this framework, protocol (10) is designed based on sampled information $p_{i}\left(t_{k}\right)$ and $q_{i}\left(t_{k}\right)$, where the lengths of the sampled periods are time-varying and can be chosen differently and arbitrarily. By contrast, in many existing works [34,38,37], there exists tight coupling among sampled periods, coupling gains and communication topologies. In this paper, the above-mentioned tight coupling is removed. That is to say, sampled periods and the coupling gains can be designed separately, which is very significant in practical applications.

Remark 2 The consensus problem by using sampled data has been discussed for second-order multi-agent systems in [34,35,37], where it is assumed that the network contains a directed spanning tree. The protocols in this paper extend the directed scenario in [34,35,37] to the directed switching topologies. It is only assumed that the union of communication topologies has a spanning tree. Besides, the interconnection topology only exists and switches at each sampled instant rather than sampled interval. Thus, it is unnecessary to 


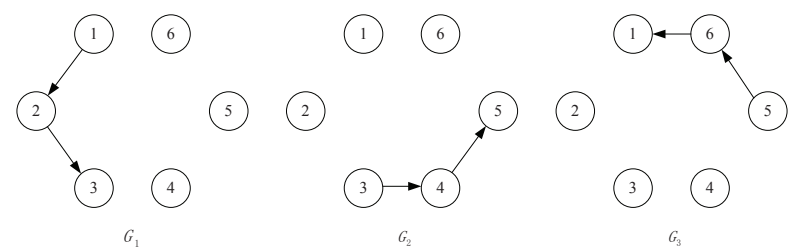

Fig. 1. The directed switching communication topology with 6 agents described by (1).

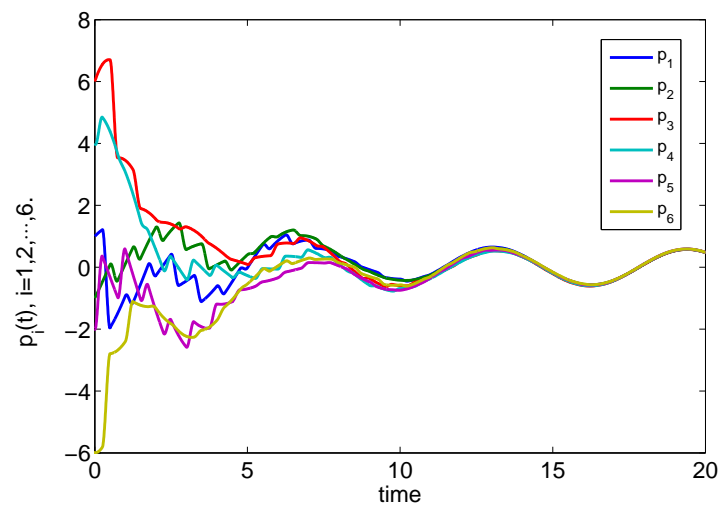

Fig. 2. The positions of multi-agent system (1) with sampled-data based algorithm (10) under directed switching topology in Fig. 1.

exchange information over the sampled interval, and the connectivity of communication topology at each sampled instant is also dispensable. These can effectively reduce the network communication.

Example 1 Consider a multi-agent system with 6 agents described by (1) with $\omega=1$. The initial states are $p_{1}(0)=1, p_{2}(0)=-1, p_{3}(0)=6, p_{4}(0)=$ $4, p_{5}(0)=-2, p_{6}(0)=-6, q_{1}(0)=1, q_{2}(0)=2, q_{3}(0)=3, q_{4}(0)=-3, q_{5}(0)=$ $-2, q_{6}(0)=0$. Choose the time series as $t_{k}=k T+t_{0}$, where $T=0.25$ and $t_{0}=0$. Assume the communication topology switches at $t_{k}$ in a random order between the three topologies shown in Figs. 1. The simulation results are shown in Fig. 2 and 3. In Fig. 2, it shows that the positions of multi-agent system (1) under protocol (10) achieve consensus. In Fig. 3, the velocities also achieve consensus.

\section{Distributed leader-following consensus for multiple harmonic os- cillators under directed switching topology.}

In this section, we study distributed leader-following consensus problems for multiple harmonic oscillators with a leader in networks. Suppose that there are $N$ followers and a leader. Without loss of generality, it is assumed that the agents labeled by $1, \cdots, N$, are followers, while the agent labeled by $N+1$ is leader. The dynamics of the leader is given by 


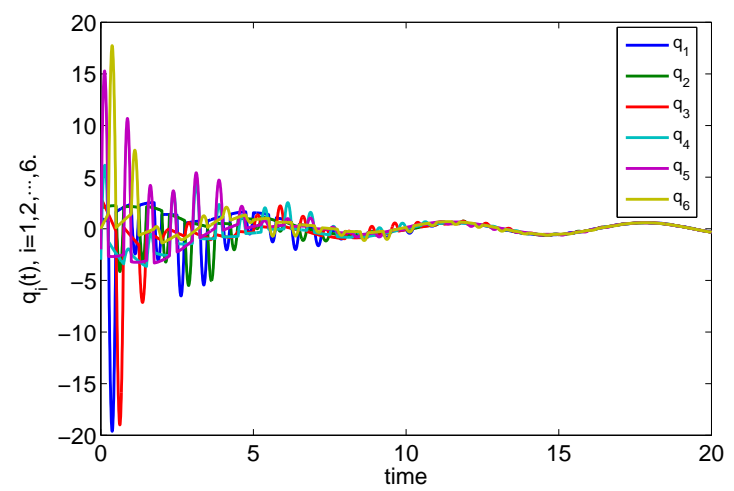

Fig. 3. The velocities of multi-agent system (1) with sampled-data based algorithm (10) under directed switching topology in Fig. 1.

$$
\left\{\begin{array}{l}
\dot{p}_{N+1}(t)=q_{N+1}(t), \\
\dot{q}_{N+1}(t)=-\omega^{2} p_{N+1}(t),
\end{array}\right.
$$

with an initial position $p_{N+1}(0)=r$ and an initial velocity $q_{N+1}(0)=v$. The dynamics of followers are given by (1). For directed switching graphs $\mathcal{G}(k)$, the associated Laplacian matrix $\mathcal{L}(k)$ can be partitioned as

$$
\mathcal{L}(k)=\left(\begin{array}{cc}
\mathcal{L}_{f}(k) & b(k) \\
0 & 0
\end{array}\right),
$$

where $\mathcal{L}_{f}(k) \in \mathbb{R}^{N \times N}$ and $b(k)=\left(b_{1}(k), b_{2}(k), \cdots\right.$, $\left.b_{N}(k)\right)^{T} \in \mathbb{R}^{N \times 1}$ is a column vector.

Throughout this section, the communication topology verifies the following assumption:

Assumption 2 For the time series $\left\{t_{k}\right\}$ with $t_{0}=0$, there exists an infinite sequence of contiguous, non-empty and uniformly bounded time intervals $\left[t_{k_{j}}, t_{k_{j+1}}\right), j=0,1, \cdots$, and $m_{j}$ switching topologies in each uniformly bounded time interval $\left[t_{k_{j}}, t_{k_{j+1}}\right)$ (i.e. there exists a finite sequence of contiguous and non-empty time subseries $t_{k_{j}}, t_{k_{j}+1}, t_{k_{j}+2}, \cdots, t_{k_{j}+m_{j}-1}$, with $t_{k_{j+1}}=t_{k_{j}+m_{j}}$, and the interconnection topologies only exist at the time instant) such that across each time interval $\left[t_{k_{j}}, t_{k_{j+1}}\right)$, for each follower, there exists a jointly directed path from the leader to that follower. In other words, for each follower, there exists a jointly directed path from the leader to that follower at the discrete times over $\left[t_{k_{j}}, t_{k_{j+1}}\right), j=0,1, \cdots$, with $t_{k_{0}}=t_{0}$.

Consider the matrix $\prod_{k=0}^{\infty}\left[I_{N}-\left(\mathcal{N}(k)+I_{N}\right)^{-1} \mathcal{L}_{f}(k)\right]$. One has the following lemma.

Lemma 2 If Assumption 2 holds, then, for the matrix (16), one has 


$$
\prod_{k=0}^{\infty}\left[I_{N}-\left(\mathcal{N}(k)+I_{N}\right)^{-1} \mathcal{L}_{f}(k)\right]=0
$$

Proof: According to Assumption 2 and Lemma 1, for the matrix (16), it follows that there exists a column vector $\xi$ such that

$$
\prod_{k=0}^{\infty}\left[I_{N+1}-\left(\mathcal{M}(k)+I_{N+1}\right)^{-1} \mathcal{L}(k)\right]=\mathbf{1} \xi^{T}
$$

where $\mathcal{M}(k)=\operatorname{diag}(\mathcal{N}(k), 0)$. Note that

$$
\begin{aligned}
& {\left[I_{N+1}-\left(\mathcal{M}(k)+I_{N+1}\right)^{-1} \mathcal{L}(k)\right] } \\
= & {\left[\begin{array}{cc}
I_{N}-\left(\mathcal{N}(k)+I_{N}\right)^{-1} \mathcal{L}_{f}(k) & -\left(\mathcal{N}(k)+I_{N}\right)^{-1} b(k) \\
0_{1 \times N} & 1
\end{array}\right] . }
\end{aligned}
$$

Thus, one has

$$
\begin{aligned}
\prod_{k=0}^{\infty}\left[I_{N+1}-\left(\mathcal{M}(k)+I_{N+1}\right)^{-1} \mathcal{L}(k)\right] & =\left[\begin{array}{cc}
\prod_{k=0}^{\infty}\left[I_{N}-\left(\mathcal{N}(k)+I_{N}\right)^{-1} \mathcal{L}_{f}(k)\right] * \\
0 & 1 \times N
\end{array}\right] \\
& =\mathbf{1} \xi^{T} .
\end{aligned}
$$

Therefore, it follows that

$$
\prod_{k=0}^{\infty}\left[I_{N}-\left(\mathcal{N}(k)+I_{N}\right)^{-1} \mathcal{L}_{f}(k)\right]=0
$$

The proof is completed.

Definition 2 For systems (1) and (15), distributed leader-following consensus problem is said to be solved if and only if there exists a distributed protocol $u_{i}(t)$, based only on the relative states information such that $\lim _{t \rightarrow \infty} \|$ $p_{i}(t)-p_{N+1}(t) \|=0$ and $\lim _{t \rightarrow \infty}\left\|q_{i}(t)-q_{N+1}(t)\right\|=0$, for $i=1,2, \cdots, N$.

Based only on the relative state information, the control goal here is to guarantee the states of the followers to converge to that of the leader. Let $\mathcal{F} \triangleq\{1, \cdots, N\}$ denote the follower set. The neighbors of agent $i$ in the follower set $\mathcal{F}$ are denoted by $\mathcal{F}_{i}=\{j \in \mathcal{F} \mid(j, i) \in \mathcal{E}\}$. Then, for the leaderfollowing consensus tracking problem concerned, the following control protocol is proposed

$$
u_{i}(t)=\frac{\alpha\left(t, t_{k}, t_{k+1}\right)}{\left|\mathcal{N}_{i}(k)\right|+1}\left[\sum_{j \in \mathcal{F}_{i}(k)} a_{i j}(k)\left[p_{j}\left(t_{k}\right)-p_{i}\left(t_{k}\right)\right]\right.
$$




$$
\begin{aligned}
& \left.+b_{i}(k)\left(p_{N+1}\left(t_{k}\right)-p_{i}\left(t_{k}\right)\right)\right] \\
& +\frac{\beta\left(t, t_{k}, t_{k+1}\right)}{\left|\mathcal{N}_{i}(k)\right|+1}\left[\sum_{j \in \mathcal{F}_{i}(k)} a_{i j}(k)\left[q_{j}\left(t_{k}\right)-q_{i}\left(t_{k}\right)\right]\right. \\
& \left.+b_{i}(k)\left(q_{N+1}\left(t_{k}\right)-q_{i}\left(t_{k}\right)\right)\right]
\end{aligned}
$$

where $b_{i}(k)$ has been defined in (16), and $\alpha\left(t, t_{k}, t_{k+1}\right)$ and $\beta\left(t, t_{k}, t_{k+1}\right)$ are given in (11).

Theorem 2 Suppose Assumption 2 holds. For the given time series $\left\{t_{k}, k=\right.$ $1,2, \cdots\}$ satisfying $0<T_{l} \leq t_{k+1}-t_{k} \leq T_{u}<\infty$, where $T_{l}$ and $T_{u}$ are two arbitrary positive constants, distributed leader-following consensus tracking problem for multi-agent systems (1) and (15) is solved by using the sampled-data based protocol (17).

Proof: By substituting (17) into (1), one gets

$$
\left\{\begin{aligned}
\dot{p}_{i}(t)= & q_{i}(t), \\
\dot{q}_{i}(t)=-\omega^{2} p_{i}(t) & +\frac{\alpha\left(t, t_{k}, t_{k+1}\right)}{\left|\mathcal{N}_{i}(k)\right|+1}\left[\sum_{j \in \mathcal{F}_{i}(k)} a_{i j}(k)\left(p_{j}\left(t_{k}\right)-p_{i}\left(t_{k}\right)\right)\right. \\
& \left.+b_{i}(k)\left(p_{N+1}\left(t_{k}\right)-p_{i}\left(t_{k}\right)\right)\right] \\
& +\frac{\beta\left(t, t_{k}, t_{k+1}\right)}{\left|\mathcal{N}_{i}(k)\right|+1}\left[\sum_{j \in \mathcal{F}_{i}(k)} a_{i j}(k)\left(q_{j}\left(t_{k}\right)-q_{i}\left(t_{k}\right)\right)\right. \\
& \left.+b_{i}(k)\left(q_{N+1}\left(t_{k}\right)-q_{i}\left(t_{k}\right)\right)\right]
\end{aligned}\right.
$$

Then, integrating (18) from $t_{k}$ to $t_{k+1}$, it is obtained that

$$
\begin{aligned}
p_{i}\left(t_{k+1}\right)= & \frac{\cos \left[\omega\left(t_{k+1}-t_{k}\right)\right]}{\left|\mathcal{N}_{i}(k)\right|+1} \cdot\left[\sum_{j \in \mathcal{F}_{i}(k)} p_{j}\left(t_{k}\right)+b_{i}(k) p_{N+1}\left(t_{k}\right)+p_{i}\left(t_{k}\right)\right] \\
& +\frac{\sin \left[\omega\left(t_{k+1}-t_{k}\right)\right]}{\omega\left|\mathcal{N}_{i}(k)\right|+1} \cdot\left[\sum_{j \in \mathcal{F}_{i}(k)} q_{j}\left(t_{k}\right)+b_{i}(k) q_{N+1}\left(t_{k}\right)+q_{i}\left(t_{k}\right)\right], \\
q_{i}\left(t_{k+1}\right)= & \frac{-\omega \sin \left[\omega\left(t_{k+1}-t_{k}\right)\right]}{\left|\mathcal{N}_{i}(k)\right|+1} \cdot\left[\sum_{j \in \mathcal{F}_{i}(k)} p_{j}\left(t_{k}\right)+b_{i}(k) p_{N+1}\left(t_{k}\right)+p_{i}\left(t_{k}\right)\right] \\
& +\frac{\cos \left[\omega\left(t_{k+1}-t_{k}\right)\right]}{\left|\mathcal{N}_{i}(k)\right|+1} \cdot\left[\sum_{j \in \mathcal{F}_{i}(k)} q_{j}\left(t_{k}\right)+b_{i}(k) q_{N+1}\left(t_{k}\right)+q_{i}\left(t_{k}\right)\right], \\
& i=1,2, \cdots, N .
\end{aligned}
$$

Further, it is followed that

$$
p_{i}\left(t_{k+1}\right)-\cos \left[\omega\left(t_{k+1}-t_{k}\right)\right] p_{i}\left(t_{k}\right)-\frac{1}{\omega} \sin \left[\omega\left(t_{k+1}-t_{k}\right)\right] q_{i}\left(t_{k}\right)
$$




$$
\begin{aligned}
& =\frac{\cos \left[\omega\left(t_{k+1}-t_{k}\right)\right]}{\left|\mathcal{N}_{i}(k)\right|+1} \\
& \cdot\left[\sum_{j \in \mathcal{F}_{i}(k)} a_{i j}(k)\left(p_{j}\left(t_{k}\right)-p_{i}\left(t_{k}\right)\right)+b_{i}(k)\left(p_{N+1}\left(t_{k}\right)-p_{i}\left(t_{k}\right)\right)\right] \\
& +\frac{\sin \left[\omega\left(t_{k+1}-t_{k}\right)\right]}{\omega\left|\mathcal{N}_{i}(k)\right|+1} \\
& \cdot\left[\sum_{j \in \mathcal{F}_{i}(k)} a_{i j}(k)\left(q_{j}\left(t_{k}\right)-q_{i}\left(t_{k}\right)\right)+b_{i}(k)\left(q_{N+1}\left(t_{k}\right)-q_{i}\left(t_{k}\right)\right)\right] \\
& q_{i}\left(t_{k+1}\right)+\omega \sin \left[\omega\left(t_{k+1}-t_{k}\right)\right] p_{i}\left(t_{k}\right)-\cos \left[\omega\left(t_{k+1}-t_{k}\right)\right] q_{i}\left(t_{k}\right) \\
& =-\frac{\omega \sin \left[\omega\left(t_{k+1}-t_{k}\right)\right]}{\left|\mathcal{N}_{i}(k)\right|+1} \\
& \cdot\left[\sum_{j \in \mathcal{F}_{i}(k)} a_{i j}(k)\left(p_{j}\left(t_{k}\right)-p_{i}\left(t_{k}\right)\right)+b_{i}(k)\left(p_{N+1}\left(t_{k}\right)-p_{i}\left(t_{k}\right)\right)\right] \\
& +\frac{\cos \left[\omega\left(t_{k+1}-t_{k}\right)\right]}{\left|\mathcal{N}_{i}(k)\right|+1} \\
& \cdot\left[\sum_{j \in \mathcal{F}_{i}(k)} a_{i j}(k)\left(q_{j}\left(t_{k}\right)-q_{i}\left(t_{k}\right)\right)+b_{i}(k)\left(q_{N+1}\left(t_{k}\right)-q_{i}\left(t_{k}\right)\right)\right] .
\end{aligned}
$$

Let $P\left(t_{k}\right)=\left(p_{1}\left(t_{k}\right), p_{2}\left(t_{k}\right), \cdots, p_{N}\left(t_{k}\right)\right)^{T}$ and $Q\left(t_{k}\right)=\left(q_{1}\left(t_{k}\right), q_{2}\left(t_{k}\right), \cdots, q_{N}\left(t_{k}\right)\right)^{T}$. Thus, it follows that

$$
\begin{aligned}
P\left(t_{k+1}\right)= & \cos \left[\omega\left(t_{k+1}-t_{k}\right)\right]\left[P\left(t_{k}\right)-\left(\mathcal{N}(k)+I_{N}\right)^{-1} \mathcal{L}_{f}(k) P\left(t_{k}\right)\right. \\
& \left.-\left(\mathcal{N}(k)+I_{N}\right)^{-1} b(k) p_{N+1}\left(t_{k}\right)\right]+\frac{1}{\omega} \sin \left[\omega\left(t_{k+1}-t_{k}\right)\right]\left[Q\left(t_{k}\right)\right. \\
& \left.-\left(\mathcal{N}(k)+I_{N}\right)^{-1} \mathcal{L}_{f}(k) Q\left(t_{k}\right)-\left(\mathcal{N}(k)+I_{N}\right)^{-1} b(k) q_{N+1}\left(t_{k}\right)\right] .
\end{aligned}
$$

Noting that the leader's position $p_{N+1}$ in $(15)$, one has

$$
p_{N+1}\left(t_{k+1}\right)=\cos \left[\omega\left(t_{k+1}-t_{k}\right)\right] p_{N+1}\left(t_{k}\right)+\frac{1}{\omega} \sin \left[\omega\left(t_{k+1}-t_{k}\right)\right] q_{N+1}\left(t_{k}\right) .
$$

By using $b(k)=-\mathcal{L}_{f}(k) \mathbf{1}_{N}$, one gets

$$
\begin{aligned}
& P\left(t_{k+1}\right)-\mathbf{1}_{N} p_{N+1}\left(t_{k+1}\right) \\
= & \cos \left[\omega\left(t_{k+1}-t_{k}\right)\right]\left[P\left(t_{k}\right)-\left(\mathcal{N}(k)+I_{N}\right)^{-1} \mathcal{L}_{f}(k) P\left(t_{k}\right)\right. \\
& \left.-\left(\mathcal{N}(k)+I_{N}\right)^{-1} b(k) p_{N+1}\left(t_{k}\right)-\mathbf{1}_{N} p_{N+1}\left(t_{k}\right)\right] \\
& +\frac{1}{\omega} \sin \left[\omega\left(t_{k+1}-t_{k}\right)\right]\left[Q\left(t_{k}\right)-\left(\mathcal{N}(k)+I_{N}\right)^{-1} \mathcal{L}_{f}(k) Q\left(t_{k}\right)\right. \\
& \left.-\left(\mathcal{N}(k)+I_{N}\right)^{-1} b(k) q_{N+1}\left(t_{k}\right)-\mathbf{1}_{N} q_{N+1}\left(t_{k}\right)\right] \\
= & \cos \left[\omega\left(t_{k+1}-t_{k}\right)\right]\left(I_{N}-\left(\mathcal{N}(k)+I_{N}\right)^{-1} \mathcal{L}_{f}(k)\right)
\end{aligned}
$$




$$
\begin{aligned}
& {\left[P\left(t_{k}\right)-\mathbf{1}_{N} p_{N+1}\left(t_{k}\right)\right]} \\
& +\frac{1}{\omega} \sin \left[\omega\left(t_{k+1}-t_{k}\right)\right]\left(I_{N}-\left(\mathcal{N}(k)+I_{N}\right)^{-1} \mathcal{L}_{f}(k)\right) \\
& {\left[Q\left(t_{k}\right)-\mathbf{1}_{N} q_{N+1}\left(t_{k}\right)\right] .}
\end{aligned}
$$

Denote $\eta_{P}\left(t_{k}\right)=P\left(t_{k}\right)-\mathbf{1}_{N} p_{N+1}\left(t_{k}\right)$ and $\eta_{Q}\left(t_{k}\right)=Q\left(t_{k}\right)-\mathbf{1}_{N} q_{N+1}\left(t_{k}\right)$. Then, one gets

$$
\begin{aligned}
\eta_{P}\left(t_{k+1}\right)= & \cos \left[\omega\left(t_{k+1}-t_{k}\right)\right]\left(I_{N}-\left(\mathcal{N}(k)+I_{N}\right)^{-1} \mathcal{L}_{f}(k)\right) \eta_{P}\left(t_{k}\right) \\
& +\frac{1}{\omega} \sin \left[\omega\left(t_{k+1}-t_{k}\right)\right]\left(I_{N}-\left(\mathcal{N}(k)+I_{N}\right)^{-1} \mathcal{L}_{f}(k)\right) \eta_{Q}\left(t_{k}\right) .
\end{aligned}
$$

Similarly, it is obtained that

$$
\begin{aligned}
\eta_{Q}\left(t_{k+1}\right)= & -\omega \sin \left[\omega\left(t_{k+1}-t_{k}\right)\right]\left(I_{N}-\left(\mathcal{N}(k)+I_{N}\right)^{-1} \mathcal{L}_{f}(k)\right) \\
& \eta_{P}\left(t_{k}\right)+\cos \left[\omega\left(t_{k+1}-t_{k}\right)\right]\left(I_{N}-\left(\mathcal{N}(k)+I_{N}\right)^{-1} \mathcal{L}_{f}(k)\right) \eta_{Q}\left(t_{k}\right) .
\end{aligned}
$$

In the matrix form, one has

$$
\begin{aligned}
\left(\begin{array}{c}
\eta_{P}\left(t_{k+1}\right) \\
\eta_{Q}\left(t_{k+1}\right)
\end{array}\right)= & \left(\begin{array}{cc}
\cos \left[\omega\left(t_{k+1}-t_{k}\right)\right] & \frac{1}{\omega} \sin \left[\omega\left(t_{k+1}-t_{k}\right)\right] \\
-\omega \sin \left[\omega\left(t_{k+1}-t_{k}\right)\right] & \cos \left[\omega\left(t_{k+1}-t_{k}\right)\right]
\end{array}\right) \\
& \otimes\left(I_{N}-\left(\mathcal{N}(k)+I_{N}\right)^{-1} \mathcal{L}_{f}(k)\right)\left(\begin{array}{c}
\eta_{P}\left(t_{k}\right) \\
\eta_{Q}\left(t_{k}\right)
\end{array}\right) \\
= & \left(\begin{array}{cc}
\cos \omega\left(t_{k+1}-t_{0}\right) & \frac{1}{\omega} \sin \omega\left(t_{k+1}-t_{0}\right) \\
-\omega \sin \omega\left(t_{k+1}-t_{0}\right) & \cos \omega\left(t_{k+1}-t_{0}\right)
\end{array}\right) \\
& \otimes \prod_{s=0}^{k}\left[I_{N}-\left(\mathcal{N}(s)+I_{N}\right)^{-1} \mathcal{L}_{f}(s)\right]\left(\begin{array}{c}
P\left(t_{0}\right) \\
Q\left(t_{0}\right)
\end{array}\right) .
\end{aligned}
$$

According to Lemma $2, \prod_{k=0}^{\infty}\left[I_{N}-\left(\mathcal{N}(k)+I_{N}\right)^{-1} \mathcal{L}_{f}(k)\right]=0$. Since each entry of

$$
\left(\begin{array}{cc}
\cos \left[\omega\left(t_{k+1}-t_{k}\right)\right] & \frac{1}{\omega} \sin \left[\omega\left(t_{k+1}-t_{k}\right)\right] \\
-\omega \sin \left[\omega\left(t_{k+1}-t_{k}\right)\right] & \cos \left[\omega\left(t_{k+1}-t_{k}\right)\right]
\end{array}\right)
$$

is bounded, then, for the above error systems (19), one gets $\lim _{k \rightarrow \infty}\left\|\eta_{P}\left(t_{k}\right)\right\|=$ $0, \lim _{k \rightarrow \infty}\left\|\eta_{Q}\left(t_{k}\right)\right\|=0$. That is to say, $\lim _{k \rightarrow \infty}\left\|P\left(t_{k}\right)-\mathbf{1}_{N} p_{N+1}\left(t_{k}\right)\right\|=0$ and $\lim _{k \rightarrow \infty}\left\|Q\left(t_{k}\right)-\mathbf{1}_{N} q_{N+1}\left(t_{k}\right)\right\|=0$. Moreover, integrating (18) from $t_{k}$ to $t$, it is obtained that

$$
p_{i}(t)=\cos \left[\omega\left(t-t_{k}\right)\right] p_{i}\left(t_{k}\right)+\frac{1}{\omega} \sin \left[\omega\left(t-t_{k}\right)\right] q_{i}\left(t_{k}\right)
$$




$$
\begin{aligned}
& +\left[\frac{\sin \left[\omega\left(t-t_{k}\right)\right]}{2 \omega^{3}}-\frac{t-t_{k}}{2 \omega^{2}} \cos \left[\omega\left(t-t_{k}\right)\right]\right] \lambda_{p_{i}}\left(t_{k}\right) \\
& -\frac{t-t_{k}}{2 \omega} \sin \left[\omega\left(t-t_{k}\right)\right] \lambda_{q_{i}}\left(t_{k}\right), t_{k} \leq t<t_{k+1},
\end{aligned}
$$

where

$$
\begin{aligned}
& \lambda_{p_{i}}\left(t_{k}\right)=\left\{\frac{2 \omega^{4}\left(t_{k+1}-t_{k}\right)}{\sin ^{2}\left[\omega\left(t_{k+1}-t_{k}\right)\right]-\omega^{2}\left(t_{k+1}-t_{k}\right)^{2}}\right. \\
& \left.+\frac{2 \omega^{3} \sin \left[\omega\left(t_{k+1}-t_{k}\right)\right] \cos \left[\omega\left(t_{k+1}-t_{k}\right)\right]}{\sin ^{2}\left[\omega\left(t_{k+1}-t_{k}\right)\right]-\omega^{2}\left(t_{k+1}-t_{k}\right)^{2}}\right\} \\
& \cdot \frac{1}{\left|\mathcal{N}_{i}(k)\right|+1}\left[\sum_{j \in \mathcal{F}_{i}(k)} a_{i j}(k)\left(p_{j}\left(t_{k}\right)-p_{i}\left(t_{k}\right)\right)\right. \\
& \left.+b_{i}(k)\left(p_{N+1}\left(t_{k}\right)-p_{i}\left(t_{k}\right)\right)\right] \\
& +\left\{\frac{2 \omega^{3} \sin ^{2}\left[\omega\left(t_{k+1}-t_{k}\right)\right]}{\sin ^{2}\left[\omega\left(t_{k+1}-t_{k}\right)\right]-\omega^{2}\left(t_{k+1}-t_{k}\right)^{2}}\right\} \\
& \cdot \frac{1}{\left|\mathcal{N}_{i}(k)\right|+1}\left[\sum_{j \in \mathcal{F}_{i}(k)} a_{i j}(k)\left(q_{j}\left(t_{k}\right)-q_{i}\left(t_{k}\right)\right)\right. \\
& \left.+b_{i}(k)\left(q_{N+1}\left(t_{k}\right)-q_{i}\left(t_{k}\right)\right)\right] \text {, } \\
& \lambda_{q_{i}}\left(t_{k}\right)=\frac{2 \omega^{3} \sin ^{2}\left[\omega\left(t_{k+1}-t_{k}\right)\right]}{\sin ^{2}\left[\omega\left(t_{k+1}-t_{k}\right)\right]-\omega^{2}\left(t_{k+1}-t_{k}\right)^{2}} \\
& \cdot \frac{1}{\left|\mathcal{N}_{i}(k)\right|+1}\left[\sum_{j \in \mathcal{F}_{i}(k)} a_{i j}(k)\left(p_{j}\left(t_{k}\right)-p_{i}\left(t_{k}\right)\right)\right. \\
& \left.+b_{i}(k)\left(p_{N+1}\left(t_{k}\right)-p_{i}\left(t_{k}\right)\right)\right] \\
& +\left\{\frac{2 \omega^{2}\left(t_{k+1}-t_{k}\right)}{\sin ^{2}\left[\omega\left(t_{k+1}-t_{k}\right)\right]-\omega^{2}\left(t_{k+1}-t_{k}\right)^{2}}\right. \\
& \left.-\frac{2 \omega \sin \left[\omega\left(t_{k+1}-t_{k}\right)\right] \cos \left[\omega\left(t_{k+1}-t_{k}\right)\right]}{\sin ^{2}\left[\omega\left(t_{k+1}-t_{k}\right)\right]-\omega^{2}\left(t_{k+1}-t_{k}\right)^{2}}\right\} \\
& \cdot \frac{1}{\left|\mathcal{N}_{i}(k)\right|+1}\left[\sum_{j \in \mathcal{F}_{i}(k)} a_{i j}(k)\left(q_{j}\left(t_{k}\right)-q_{i}\left(t_{k}\right)\right)\right. \\
& \left.+b_{i}(k)\left(q_{N+1}\left(t_{k}\right)-q_{i}\left(t_{k}\right)\right)\right] \text {. }
\end{aligned}
$$

Integrating (15) from $t_{k}$ to $t$, one gets

$$
\begin{aligned}
p_{N+1}(t)= & \cos \left[\omega\left(t-t_{k}\right)\right] p_{N+1}\left(t_{k}\right) \\
& +\frac{1}{\omega} \sin \left[\omega\left(t-t_{k}\right)\right] q_{N+1}\left(t_{k}\right), t_{k} \leq t<t_{k+1} .
\end{aligned}
$$


Thus,

$$
\begin{aligned}
P(t)-\mathbf{1}_{N} p_{N+1}(t)= & \cos \left[\omega\left(t-t_{k}\right)\right]\left[P\left(t_{k}\right)-\mathbf{1}_{N} p_{N+1}\left(t_{k}\right)\right] \\
& +\frac{1}{\omega} \sin \left[\omega\left(t-t_{k}\right)\right]\left[Q\left(t_{k}\right)-\mathbf{1}_{N} q_{N+1}\left(t_{k}\right)\right] \\
& +\left[\frac{\sin \left[\omega\left(t-t_{k}\right)\right]}{2 \omega^{3}}-\frac{t-t_{k}}{2 \omega^{2}} \cos \left[\omega\left(t-t_{k}\right)\right]\right] \Lambda_{P}\left(t_{k}\right) \\
& -\frac{t-t_{k}}{2 \omega} \sin \left[\omega\left(t-t_{k}\right)\right] \Lambda_{Q}\left(t_{k}\right),
\end{aligned}
$$

where $\Lambda_{P}\left(t_{k}\right)=\left(\lambda_{p_{1}}\left(t_{k}\right), \cdots, \lambda_{p_{N}}\left(t_{k}\right)\right)^{T}$ and $\Lambda_{Q}\left(t_{k}\right)=\left(\lambda_{q_{1}}\left(t_{k}\right), \cdots, \lambda_{q_{N}}\left(t_{k}\right)\right)^{T}$. Then, one has

$$
\begin{aligned}
& \left\|P(t)-\mathbf{1}_{N} p_{N+1}(t)\right\| \\
\leq & \left\|P\left(t_{k}\right)-\mathbf{1}_{N} p_{N+1}\left(t_{k}\right)\right\|+\frac{1}{\omega}\left\|Q\left(t_{k}\right)-\mathbf{1}_{N} q_{N+1}\left(t_{k}\right)\right\| \\
& +\frac{1+\left[\omega\left(t_{k+1}-t_{k}\right)\right]}{2 \omega^{3}}\left\|\Lambda_{P}\left(t_{k}\right)\right\| \\
& +\frac{t_{k+1}-t_{k}}{2 \omega}\left\|\Lambda_{Q}\left(t_{k}\right)\right\|, t_{k} \leq t<t_{k+1} .
\end{aligned}
$$

By noting that

$$
\begin{aligned}
\Lambda_{P}\left(t_{k}\right)= & \frac{2 \omega^{4}\left(t_{k+1}-t_{k}\right)+2 \omega^{3} \sin \left[\omega\left(t_{k+1}-t_{k}\right)\right] \cos \left[\omega\left(t_{k+1}-t_{k}\right)\right]}{\left[\sin ^{2}\left[\omega\left(t_{k+1}-t_{k}\right)\right]-\omega^{2}\left(t_{k+1}-t_{k}\right)^{2}\right]} \\
& \left(N(k)+I_{N}\right)^{-1}\left[-\mathcal{L}_{f}(k) P\left(t_{k}\right)-b(k) p_{N+1}\left(t_{k}\right)\right] \\
& +\frac{2 \omega^{3} \sin ^{2}\left[\omega\left(t_{k+1}-t_{k}\right)\right]}{\left[\sin ^{2}\left[\omega\left(t_{k+1}-t_{k}\right)\right]-\omega^{2}\left(t_{k+1}-t_{k}\right)^{2}\right]} \\
& \left(N(k)+I_{N}\right)^{-1}\left[-\mathcal{L}_{f}(k) Q\left(t_{k}\right)-b(k) q_{N+1}\left(t_{k}\right)\right]
\end{aligned}
$$

one has

$$
\begin{aligned}
\left\|\Lambda_{P}\left(t_{k}\right)\right\| \leq & \frac{2 \omega^{4}\left(t_{k+1}-t_{k}\right)+2 \omega^{3}}{\left[\omega^{2}\left(t_{k+1}-t_{k}\right)^{2}-\sin ^{2}\left[\omega\left(t_{k+1}-t_{k}\right)\right]\right]} \\
& \left\|\mathcal{L}_{f}(k)\right\|\left\|P\left(t_{k}\right)-\mathbf{1}_{N} p_{N+1}\left(t_{k}\right)\right\| \\
& +\frac{2 \omega^{3}}{\left[\omega^{2}\left(t_{k+1}-t_{k}\right)^{2}-\sin ^{2}\left[\omega\left(t_{k+1}-t_{k}\right)\right]\right]} \\
& \left\|\mathcal{L}_{f}(k)\right\|\left\|Q\left(t_{k}\right)-\mathbf{1}_{N} q_{N+1}\left(t_{k}\right)\right\| .
\end{aligned}
$$

Considering $\lim _{k \rightarrow \infty}\left\|P\left(t_{k}\right)-\mathbf{1}_{N} p_{N+1}\left(t_{k}\right)\right\|=0$ and $\lim _{k \rightarrow \infty}\left\|Q\left(t_{k}\right)-\mathbf{1}_{N} q_{N+1}\left(t_{k}\right)\right\|=$ 0 , one has $\lim _{k \rightarrow \infty}\left\|\Lambda_{P}\left(t_{k}\right)\right\|=0$. Similarly, $\lim _{k \rightarrow \infty}\left\|\Lambda_{Q}\left(t_{k}\right)\right\|=0$. Therefore, $\lim _{t \rightarrow \infty}\left\|P(t)-\mathbf{1}_{N} p_{N+1}(t)\right\|=0$. By the similar derivations, one gets $\lim _{t \rightarrow \infty}\left\|Q(t)-\mathbf{1}_{N} q_{N+1}(t)\right\|=0$. Thus, under the protocol (17), the states of 


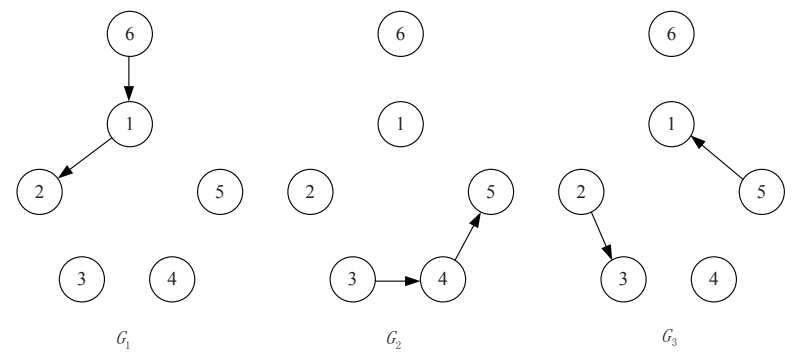

Fig. 4. The directed switching communication topology with 5 followers and 1 leader described by (1) and (15), respectively.

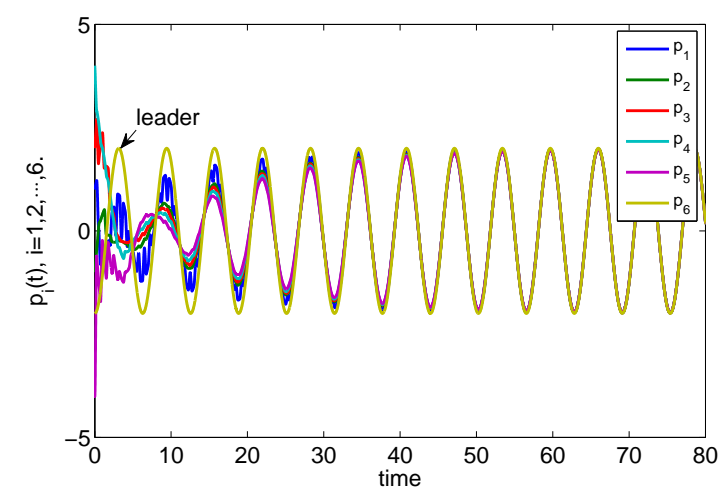

Fig. 5. The positions of the leader-following multi-agent system given by (1) and (15) under protocol (17).

the followers in systems (1) can track those of the leader in (15). The proof is completed.

Remark 3 Compared with leader-following consensus in [38], this paper extends the directed communication topology in [38] to the directed switching topologies. Furthermore, by specifying desired separations among different agents, the leader-following consensus algorithms (17) can be applied to achieve distributed coordination including formation control and flocking. For the case with multiple leaders in systems, the motion planning approaches proposed in this paper can also be used to design the containment control protocols.

Example 2 Consider a multi-agent system with 5 followers described by (1), and 1 leader described by (15) with $\omega=1$. The initial states are $p_{1}(0)=$ $1, p_{2}(0)=-1, p_{3}(0)=2, p_{4}(0)=4, p_{5}(0)=-4, p_{6}(0)=-2, q_{1}(0)=1, q_{2}(0)=$ $2, q_{3}(0)=3, q_{4}(0)=-3, q_{5}(0)=-2, q_{6}(0)=0$. Choose the time series as $t_{k}=k T+t_{0}$, where $T=0.25$ and $t_{0}=0$. Assume the directed communication topologies switch at $t_{k}$ in a random order between the three topologies shown in Fig. 4. The simulation results are shown in Figs. 5 and 6. In Fig. 5, it shows that the positions of the followers given by (1) can track the position of the leader in (15) under protocol (17). In Fig. 6, the velocities of followers also achieve the leader's velocity. 


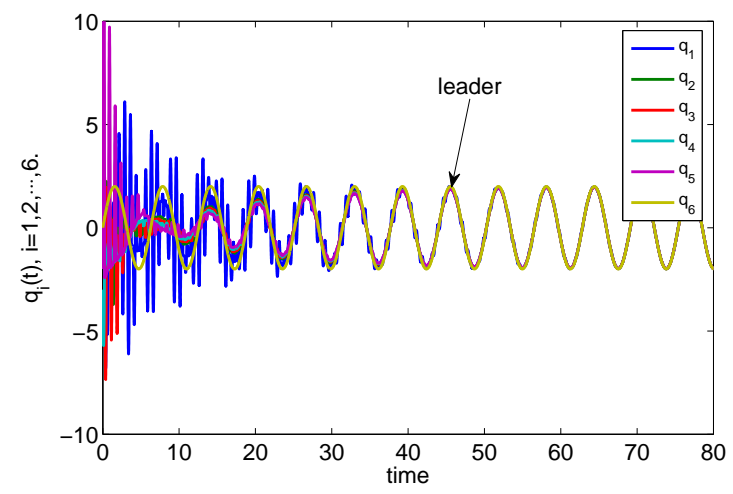

Fig. 6. The velocities of leader-following multi-agent system given by (1) and (15) under protocol (17).

\section{Conclusion}

In this paper, the distributed sampled-data based consensus problem has been investigated for a group of harmonic oscillators with directed switching topologies by using motion planning approaches. With the assumption that the union of the directed interaction graphs has a spanning tree, a class of leadless consensus protocols are designed for agents to achieve consensus. As an extension of the leaderless consensus, the leader-following consensus problem is also solved in this paper. A remarkable advantage of the proposed sampled-data based protocols is that the sampled periods, communication topologies and control gains are decoupling and can be separately designed, which relaxes many restrictions in controller designs. Future works will focus on solving distributed consensus problem for mobile agents modeled by general linear or nonlinear dynamics under directed switching topologies.

\section{References}

[1] A. Jadbabaie, J. Lin and A. S. Morse. Coordination of groups of mobile autonomous agents using nearest neighbor rules. IEEE Transactions on Automatic Control, 48(6):988-1001, 2003.

[2] R. Olfati-Saber and R. M. Murray. Consensus problems in networks of agents with switching topology and time-delays. IEEE Transactions on Automatic Control, 49(9):1520-1533, 2004.

[3] W. Ren and R. W. Beard. Consensus seeking in multiagent systems under dynamically changing interaction topologies. IEEE Transactions on Automatic Control, 50(5):655-661, 2005.

[4] Y. Z. Lv, Z. K. Li, Z. S. Duan, and J. Chen. Distributed adaptive output feedback consensus protocols for linear systems on directed graphs with a leader of bounded input, Automatica, 74:308-314, 2016. 
[5] Z. K. Li, M. Chen, and Z. T. Ding. Distributed adaptive controllers for cooperative output regulation of heterogeneous agents over directed graphs, Automatica, 68:179-183, 2016.

[6] Y. Zhao, Z. S. Duan, G. H. Wen, and G. R. Chen. Distributed average computation for multiple time-varying signals with output measurements, International Journal of Robust and Nonlinear Control, 26(13):2899-2915, 2016.

[7] Y. Zhao, Y. F. Liu, Z. K. Li, and Z. S. Duan. Distributed average tracking for multiple signals generated by linear dynamical systems: an edge-based framework, Automatica, 75:158-166, 2017.

[8] J. Y. Sun, Z. Y. Geng and Y. Z. Lv. Adaptive output feedback consensus tracking for heterogeneous multi-agent systems with unknown dynamics under directed graphs, System \& Control Letters, 87:16-22, 2016.

[9] N. Huang, Z. S. Duan and Y. Zhao. Distributed consensus for multiple Euler-Lagrange systems: An event-triggered approach, SCIENCE CHINA: Technological Sciences, 59(1), 33-44, 2016.

[10] J. Y. Wang, Z. S. Duan, G. H. Wen, and G. R. Chen. Distributed robust control of uncertain linear multi-agent systems, International Journal of Robust and Nonlinear Control, 25(13), 2162-2179, 2015.

[11] B. H. Wang, J. C. Wang, B. Zhang, W. S. Chen, and Z. Q. Zhang. Leaderfollower consensus of multi-vehicle wirelessly networked uncertain systems subject to nonlinear dynamics and actuator fault, IEEE Transactions on Automation Science and Engineering, accepted in press, doi:10.1109/TASE.2016.2635979, 2016.

[12] X. Y. Liu, J. D. Cao, W. W. Yu, and Q. Song. Nonsmooth finite-time synchronization of switched coupled neural networks, IEEE Transactions on Cybernetics, 46(10):2360-2371, 2016.

[13] X. Y. Liu, D. Ho, J. D. Cao, and W. Y. Xu. Discontinuous observers design for finite-time consensus of multiagent systems with external disturbances, IEEE Transactions on Neural Networks and Learning Systems, accepted in press, DOI: 10.1109/TNNLS.2016.2599199, 2016.

[14] P. Lin, M. X. Dai and Y. D. Song. Consensus stability of a class of secondorder multi-agent systems with nonuniform time-delays, Journal of The Franklin Institute, 351(3):1571-1576, 2014.

[15] J. J. Fu and J. Z. Wang. Output consensus of heterogeneous linear systems with quantized information, Journal of Franklin Institute, 351(3):1400-1418, 2014.

[16] Y. F. Liu, Y. Zhao, and G. R. Chen. Finite-time formation tracking control for multiple vehicles: A motion planning approach, International Journal of Robust and Nonlinear Control, 26(14):3130-3149, 2016.

[17] Y. F. Liu, and Z. Y. Geng. Finite-time formation control for linear multi-agent systems: A motion planning approach, Systems \& Control Letters, 85(11):64-60, 2015.

[18] Y. F. Liu, Y. Zhao, and G. R. Chen. Sampled-data-based consensus and containment control of multiple harmonic oscillators: A motion-planning approach, Chaos, 26(11):116303, 2016. 
[19] Y. Zhao, and Z. S. Duan. Finite-time containment control without velocity and acceleration measurements, Nonlinear Dynamics, 82(1-2):259-268, 2015.

[20] Y. F. Liu, Y. Zhao, Z. K. Shi, and D. M. Wei. Specifed-time containment control of multi-agent systems over directed topologies, IET Control Theory \& Applications, accepted in press DOI:10.1049/iet-cta.2016.1159, 2016.

[21] J. J. Fu and J. Z. Wang. Robust finite-time containment control of general linear multi-agent systems under directed communication graphs, Journal of Franklin Institute, 353(12):2670-2689, 2016.

[22] X. W. Dong, Q. D. Li, Z. Ren and Y. S. Zhong. Formation-containment control for high-order linear time-invariant multiagent systems with time delays, Journal of Franklin Institute, 352(9):3564-3584, 2015.

[23] X. W. Dong, Y. Zhou, Z. Ren and Y. S. Zhong. Time-varying formation control for general linear multi-agent systems with switching directed topologies, Control Engineering Practice, 46: 26-36, 2016.

[24] X. W. Dong, Y. Zhou, Z. Ren and Y. S. Zhong. Time-varying formation tracking for second-order multi-agent systems subjected to switching topologies with application to quadrotor formation flying, IEEE Transactions on Industrial Electronics, accepted in press, DOI: 10.1109/TIE.2016.2593656, 2016.

[25] X. W. Dong and G. Q. Hu. Time-varying formation control for general linear multi-agent systems with switching directed topologies, Automatica, 73:47-55, 2016.

[26] Q. Song, J. D. Cao and W. W. Yu. Second-order leader-following consensus for nonlinear multi-agent systems via pinning control, Systems \& Control Letters, $59(9), 553-562,2010$.

[27] Q. Song, F. Liu, G. H. Wen, J. D. Cao, and Y. Tang. Synchronization of coupled harmonic oscillators via sampled position data control, IEEE Transactions on Circuits and Systems I: Regular Papers, 63(7), 1079-1088, 2016.

[28] W. B. Zhang, Y. Tang, T. W. Huang, and J. Kurths. Sampled-data consensus of linear multi-agent systems with packet losses, IEEE Transactions on Neural Networks and Learning Systems, 63(7), 1079-1088, 2016.

[29] Y. Zhao, Z. S. Duan, and G. H. Wen. Distributed finite-time tracking of multiple Euler-Lagrange systems without velocity measurements. International Journal of Robust and Nonlinear Control, 25(11):1688-1703, 2015.

[30] Y. Zhao, Z. S. Duan, and G. H. Wen. Distributed finite-time tracking for a multi-agent system under a leader with bounded unknown acceleration, Systems \& Control Letters 81(7):8-13, 2015.

[31] Y. Zhao, Z. S. Duan, G. H. Wen and G. R. Chen. Distributed finite-time tracking of multiple non-identical second-order nonlinear systems with settling time estimation, Automatica, 64(2):86-93, 2016.

[32] G. M. Xie, H. Y. Liu, L. Wang and Y. M. Jia. Consensus in networked multiagent systems via sampled control: fixed topology case, Preceedings of 2009 American Control Conference, St. Louis, Missouri, 3902-3907, 2009. 
[33] Y. P. Gao and L. Wang. Sampled-data based consensus of continuous time multiagent systems with time-varying topology. IEEE Transactions on Automatic Control, 56(5):1226-1231, 2011.

[34] Y. C. Cao and W. Ren. Multi-vehicle coordination for double-integrator dynamics under fixed undirected/directed interaction in a sampled-data setting, International Journal of Robust and Nonlinear Control, 20(9):987-1000, 2010.

[35] W. W. Yu, W. X. Zheng, G. R. Chen, W. Ren and J. D. Cao. Secondorder consensus in multi-agent dynamical systems with sampled position data, Automatica, 47(7):1496-1503, 2011.

[36] Q. Ma, S. Y. Xu and F. Lewis. Second-order consensus for directed multiagent systems with sampled data, International Journal of Robust and Nonlinear Control, 24(16):2560-2573, 2013.

[37] N. Huang, Z. S. Duan and G. R. Chen. Some necessary and sufficient conditions for consensus of second-order multi-agent systems with sampled position data, Automatica 63(1):148-155, 2016.

[38] G. H. Wen, Z. S. Duan, W. W. Yu and G. R. Chen. Consensus of multi-agent systems with nonlinear dynamics and sampled-data information: a delayed-input approach, International Journal of Robust and Nonlinear Control, 23(6):602-619, 2013.

[39] A. E. Bryson and Y. C. Ho. Applied optimal control, Hemisphere Publishing Corporation London, 1975. 